Zabytkoznawstwo i Konserwatorstwo XIII, Torun 2011

Viola Hildebrand-Schat

Goethe-Universität Frankfurt am Main

\title{
Die Danziger Gebote-Tafeln als Spiegel ihrer Zeit
}

, $\begin{aligned} & \text { ie Marienkirche ist nicht nur die Zierde aller Danziger Kirchen, } \\ & \text { sondern wird mit Recht die Krone dieser Stadt genannt." }\end{aligned}$ dieser Feststellung liefert der Herausgeber der mehrbändigen Reihe Bauund Kunstdenkmäler des deutschen Ostens nur das Resümee einer jahrhundertelangen Beobachtung von Danzig-Reisenden. Die Marienkirche ist aufgrund ihrer reichen Ausstattung an Epitaphen, Bildwerken und Altären praktisch seit ihrem Bestehen für Gläubige wie Kun streisende ein beliebter Anlaufpunkt. Die noch heute reiche und durchweg gut erhaltene Ausstattung der Kirche läßt nichts oder doch nur wenig von ihrer bewegten Geschichte ahnen, die sie zum Schauplatz verschiedener Konfessionen werden ließ und - wie viele andere Kirchenbauten auch - den nachreformatorischen Bilderstürmen aussetzte.

Erwähnenswert sind neben vielem anderen ${ }^{2}$ zwei großformatige Tafeln, die den Kirchenbesucher an seine Pflichten als Christ erinnern sollen.

1 Günther Grundmann an Willi Drost in einem Schreiben vom 10. September 1962, in: W. Drost, Die Marienkirche in Danzig und ibre Kunstschätze, Stuttgart 1962, S. 5.

2 Allgemein wird auf die Astronomische Uhr und vor allem das Weltgerichtstriptychon von Hans Memling hingewiesen, letzteres nur mehr als Kopie in der Kirche vorhanden. Das Original befindet sich im Nationalmuseum in Danzig. 
Nur durch einen Vierungspfeiler voneinander getrennt, präsentieren sich eine zwischen 1480 und 1490, wahrscheinlich von einem niederdeutschen Meister, geschaffene Darstellung der Zehn Gebote und ein als „Almosentafel“" bezeichnetes Gemälde von Anton Möller aus dem Jahre 1607.3

Beide Tafeln lassen sich nicht einfach unter die Kategorie „Andachtsbild" subsumieren, will man vermeiden, den Begriff der Andacht undifferenziert auf alles zu beziehen, was im weitesten Sinne Umgang mit geistlichen Bildinhalten ist. Passend erscheint hingegen, Ruth Slenczka folgend, die Bezeichnung als „lehrhafte Bildtafel.“4 (Beichttafel oder Predigttafel, Hervé Martin) Solche Bilder waren kein Gegenstand der Verehrung, vielmehr sollten sie dem Betrachter als Hinweis dienen, worauf er seine Aufmerksamkeit zu richten hatte und so eine der Schrift vergleichbare Aufgabe übernehmen. Gemälde wurden gemeinhin als Bücher der Laien gesehen, wobei mit der Bezeichnung „Laien“ nicht nur die des Lesens Unkundigen gemeint waren, sondern generell alle Nichtgeistlichen. Damit erklärt sich auch, daß den Bildern häufig volkssprachliche Beschriftungen beigefügt waren. ${ }^{5}$

Thematisch verwandt - beide Tafeln richten sich mit Anweisungen an ihre Betrachter - unterscheiden sich die Darstellungen entsprechend ihrer Entstehungszeit formal wie auch inhaltlich. Ist erstere noch ganz einer spätmittelalterlichen Auffassung verhaftet, die das Bildgeschehen durch Spruchbänder unterstreicht, neigt letztere einer barockisierenden Sicht zu, in der sich mehrere Bildebenen sowie ikongraphische Muster überlagern und durchdringen. Die Tafel mit den Zehn Geboten ist vorreformatorisch, ${ }^{6}$ die Almosentafel mit den Werken der Barmherzigkeit nachreformatorisch. Beide suchen die ihrer Zeit gemäße Lehre zu vermit-

3 Offensichtlich hat die Tafel ihren Platz mehrfach geändert, Frisch sieht sie an jenem o. e. Pfeiler der Vierung verortet, dem Dissertation Spätgotische Tafelmalerei in Danzig (Erlangen 1937) von Werner Kussin beigelegten Plan ist allerdings zu entnehmen, daß sie vor 1937 in der dritten Kappel rechts im Kappellenkranz um den Hochaltar angebracht war.

4 R. Slenczka, Lehrbafte Bildtafeln in spätmittelalterlichen Kirchen, Köln-Weimar-Wien 1998, S. 13.

5 Ibidem, S. 15.

6 Schultz bezeichnet sie als das älteste Gemälde der Marienkirche überhaupt. Vgl. Schultz, Über altertümliche Gegenstände der bildenden Kunst in Dan₹ig, 1841. 
teln, doch nehmen beide darüber hinaus insofern in besondere Weise gefangen, als sie sich mit einem unmittelbaren sozialgeschichtlichen Bezug an den Betrachter wenden. Deutlich wird dies am motivischen Repertoire, mit dem die Tafel der Zehn Gebote auf Landschaft und Bevölkerung der Stadt Danzig eingeht, die Almosentafel hingegen auf die religiösen Konstellationen, die um $1600 \mathrm{zu}$ miteinander konkurrierenden Mächten ausgewachsen waren und um ihre Vormachtstellung stritten.

\section{Die Tafel der Zehn Gebote*}

Am nordwestlichen freistehenden Vierungspfeiler, also am Ende des Langhauses und unweit vom Chor, befindet sich - weit über Kopfhöhe angebracht - die Bildtafel mit den zehn Geboten. Aufgrund der Höhe, in der sich das Gemälde befindet, ist ein Betrachten nur mit einigem Abstand möglich und in jedem Fall der Betrachter gezwungen, seinen Blick zu heben. Das gesamte etwa 2 mal 3,5 Meter große Bildfeld ist durch eine mittlere Senkrechte zweigeteilt und in zehn annähernd gleich große Felder aufgegliedert $(61 \times 10,2 \mathrm{~cm})$, wobei die beiden nach oben abschließenden ein Bogenfeld bilden und dadurch im Format abweichen. Dargestellt sind in Wort und Bild die Zehn Gebote, wobei links die Einhaltung, rechts die Übertretung umgesetzt ist, kenntlich gemacht durch Engel und Teufel, die den jeweiligen Gruppen beigeordnet sind. Der zugehörige Text findet sich sowohl auf den querlaufenden Rahmenleisten wie auch auf den in die Bilder integrierten Spruchbändern? ${ }^{7}$. Wiedergegeben ist der Wort-

\footnotetext{
* Am nordwestlichen Vierungspfeiler nach Südosten aufgehängt, Holz, $251 \times$ $\times 205 \mathrm{~cm}$ mit lünettenförmigem Aufsatz, dessen größte Höhe 95,5 cm ist; Format der einzelnen 10 Felder (Bogenfelder mit eingeschlossen) ca. $61 \times 10,2 \mathrm{~cm}$.

7 Eine sorgfältige Wiedergabe der Inschriften liefert Gregorius Frisch: Der Sankt Marienpfarrkirche in Dantzig inwendige Abriß. Beschreibung der Oberpfarrkirche zu Sankt Marien in Danzig und der inneren Merkwürdigkeiten derselben, vorzüglich des berühmten Altargemäldes, auf welchem das Jüngste Gericht abgebildet ist (1698), hrsg. von K. Cieślak, Danzig (Officina Ferberiana) 1999, S. 102-104. Die Handschrift besteht aus 114 Papierblättern im Format $19 \times 16 \mathrm{~cm}$, die mit Ausnahme von je zwei, die während einer Restaurierung vor und nach dem Krieg eingeklebt wurden, original sind. Frisch war Küster in St. Marien und hat ein genaues Inventar der Epitaphien und Altäre zu-
} 
laut der Gebote - allerdings in einer ausführlicheren Version als auf der Randleiste, bisweilen aber auch die Empfindungen der Frevelnden oder die Beschwichtigungen der Teufel. Gleichzeitig werden hier auch die Gegenargumente vorgebracht. So heißt es im ersten Bildfeld: „Du sollst anbeten einen Gott,“ dem dann im zweiten Spruchband auf der rechten Bildseite im linken Bildfeld gegenübergestellt ist: „Ach, was hast Du zu schaffen, laß beten Mönche und Pfaffen." Im rechten Bildfeld folgt dann das zweite Gebot, daß auf der Rahmenleiste mit „Dies ist das andre Gebot, du sollst durch Gott nicht schwören" eingeführt wird, um in dem einen Spruchband innerhalb des Bildes wiederum aufgegriffen und im zweiten mit „Dir ist gering zu schwören, ich lasse dich nicht umkehren“ widerlegt zu werden. ${ }^{8}$ In dieser Weise werden alle weiteren Gebote vorgetragen, wobei die Bildfelder von Gebot und Widerspruch teilweise durch architektonische Elemente voneinander abgetrennt, teilweise aber auch übergangslos miteinander verbunden sind, gleichsam um zu demonstrieren, wie leicht die Gebote übertreten werden können. Weiterhin fällt auf, daß der wiedergegebene Wortlaut mit keiner der beiden zentralen Bibelstellen - weder mit dem 2. Buch Moses 20, 2-17 noch dem 5. Buch Moses 5, 6-21 - übereinstimmt.

Der Spruch auf der unteren Abschlußleiste liefert dann eine Zusammenfassung der mit den Geboten intendierten Aussage: links steht, Matthäus 19,7 zitierend, in deutlich größeren Buchstaben als der übrige Text: „Si vis ad vitam ingredi serva mandata“ (Wenn du zum Leben gelangen willst, halte dich an die Gebote/bewahre das dir anvertraute Gesetz), gefolgt von einer volkssprachlichen deutschen Umschreibung der Aussage: „Hier stehen gemalt die zehn Gebote, die sollst du rücken eben, die halt wie Gold, das ist dir not, willst du ewig leben." Nicht nur sind die la-

sammengestellt. Biblioteka Gdańska Polskiej Akademii Nauk, Ms. Mar. Q 146. Über Frisch findet sich ein knapper Eintrag im Steinbuch der Marienkirche im Staatsarchiv Danzig, 354/347, Fol. 245v-246r: „Gregorius Frysch, um 1640 geboren, verstorben im Alter von 69 Jahren, am 30. Dezember 1709 beerdigt. Er war verheiratet: seine mit 63 Jahren verstorbene Ehefrau wurde an gleicher Stelle am 15. September 1700 begraben."

8 Wiedergegeben ist die Schriftsprache des ostmitteldeutschen Raumes, wie sie der Deutsche Orden, aber auch die Danziger Kanzleien vor allem im 15. Jahrhundert anwandten (laut Kussin, der Hans J. Schneider vom Deutschen Sprachatlas zitiert). 
teinischen Worte auffallend hervorgehoben, sondern, da sie sich zudem am unteren Rand befinden, wirklich auch für jedermann gut erkennbar. Damit erhalten sie besonderen Nachdruck, der ihre herausgehobene Bedeutung unterstreicht.

Alles in allem entsprechen die Darstellungen dem, was als Grundlage der Beichte zu dienen hatte, orientierte sich doch das seit der Mitte des 15. Jahrhunderts geläufigste Beichtschema an den Zehn Geboten. Vor diesem Hintergrund erklärt sich auch die Bedeutung des Gemäldes als Beichttafel. Die Beichte war zentrales Anliegen der spätmittelalterlichen Laienunterweisung. Spätestens seit Einführung des Pflichtberichts im vierten Laterankonzil 1215 war jeder Christ dazu aufgefordert, mindestens einmal im Jahr einen Priester aufzusuchen, um seine Sünden darzulegen. Zur Vorbereitung dienten einerseits Beichttraktate, andererseits aber auch der Gottesdienst, bei dem die notwendigen Regeln eingeübt werden sollten. Der Mainzer Erzbischof Berthold von Henneberg verlieh 1493 allen Priestern 40 Tage Ablaß, wenn sie in ihren Sonn- und Feiertagsgottesdiensten der Gemeinde das Pater noster, das Ave Maria, das apostolische Glaubensbekenntnis und den Dekalog Wort für Wort vorsprachen. Unterstützend wirkten hierbei Schrift- und Bildtafeln, wie eben jene Danziger Tafel mit den Zehn Geboten. Inhaltlich und kompositorisch mnemotechnisch durchdacht, lieferten sie eine gute Gedankenstütze und waren als Lernhilfe geeignet. Die Bedeutung, die ihnen zugemessen wurde, ermißt sich aus der Vorrede, die der Straßburger Stadtprediger Geiler von Kaysersberg $1510 \mathrm{zu}$ Johannes Gersons katechetischer Schrift Opus tripartitum verfaßte. Hier fordert er ausdrücklich dazu auf, Tafeln solcher Art in allen Kirchen anzubringen. Sein Vorschlag erreichte eine breite Leserschaft und an verschiedenen Orten wurde er auch ohne obrigkeitliche Anweisung umgesetzt. Die Danziger Tafel fügt sich insofern vollständig in das Schema von Beichttafeln, als sie in ihrer Darstellung die Zehn Gebote mit den Todsünden verknüpft und somit zentrale Themen des Beichtunterrichts abdeckt (Glaubensbekenntnis, Sakramente, 10 Gebote, Todsünden, 7 Werke der Barmherzigkeit).

Den Rahmen des Geschehens bildet die Alltagswelt des Bürgertums, wobei Anspielungen auf biblische Szenen nicht fehlen. $\mathrm{Zu}$ sehen ist im oberen linken Bildfeld der Tanz um das goldenen Kalb, die Übergabe der Gesetzestafeln und ihre Präsentation durch Moses vor dem Volk, rechts 
die Mannalese. Nicht weniger explizit ausformuliert sind Regeln für Gebet, Andacht und Mahlzeiten. Die alltägliche Gegenwart findet sich in der genrehaften Wiedergabe von Innen- und Außenraum. Eine ersichtliche Zuordnung von guten und schlechten Taten zu einem spezifischen Ambiente erfolgt dabei nicht. Wohl aber scheint es naheliegend, daß der Maler einen unmittelbaren Bezug zur Stadt Danzig intendierte. Die wiederholt auftauchenden Türme und größeren Gebäudekomplexe im Hintergrund können Verweise auf die vielen Mitte des 15. Jahrhunderts bestehenden Kirchen Danzigs sein. Vor allem aber spiegeln die überwiegend in reicher Kleidung dargestellten Personen den allgemeinen Wohlstand wider, der im 15. Jahrhundert in Danzig das Leben prägte.

Nachdem Danzig 1343 das Stadtrecht erhalten hatte, erlebte die Stadt einen permanenten wirtschaftlichen Aufschwung und entwickelte sich zu einer der größten Hafenstädte Europas. Im Laufe des 15. Jahrhunderts emanzipierte sie sich schließlich weitgehend von der Hansepolitik, an der sie seit dem Ende des 13. Jahrhunderts mit einer starken Position beteiligt war, trat dem gegen die Herrschaft des Deutschen Ordens gerichteten Preußischen Bund bei, unterstützte im dreizehnjährigen Krieg (1454-1466) Polen gegen den Deutschen Orden und ging kurz darauf (1454) mit König Casimir IV. eine Schutzbeziehung ein, was bedeutete, das die Stadt von nun an dem Schutz der Krone Polens unterstand. Das brachte eine Reihe von bedeutenden Privilegien und Hoheitsrechte mit sich, wie vollständige Gerichtsbarkeit und Münzrecht, weiterhin die Befreiung von Zöllen und Abgaben sowie von der Rechnungslegung seiner Einkünfte. Festgeschrieben wurden diese Rechte im Privilegium Casimirianum. ${ }^{9}$ Auf Reichstagen stimmte die Stadt bei den Königswahlen mit und ein Mit-

9 Danzig (Mittelalter: Gedanum), wird 997 erstmals als Gyddanze, urkundlich 1148 als Kdanze als Hauptort des Herzogtums Pomerellens erwähnt. Kurz nach 1263 erhielt es Lübisches, 1343 Culmisches (Magdeburger) Stadtrecht. 1309 ging Danzig an den Deutschen Orden, 1361 wurde es Mitglied der Hanse. Durch das Privilegium Casimirianum wurde Danzig eine Freie Stadt, der polnische König besaß seit 1454 nur geringe Hoheitsrechte. 1523-1557 nahm Danzig die Reformation an. Erste Teilung Polens 1772: Danzig blieb Freie Stadt. Zweite Teilung Polens (1793): Danzig fiel an Preußen. Nach dem 1. Weltkrieg wurde Danzig mit Umgebung durch den Versailler Vertrag vom Deutschen Reich losgelöst und zur Freien Stadt erklärt. Danzig hatte u.a. die Aufgabe, Polen den freien Zugang zum Meer zu verschaffen. 
glied des Stadtrates repräsentierte die Oberhoheit des polnischen Königs. Die zahlreichen Privilegien und Schenkungen bildeten die Basis für die besondere Stellung der Stadt innerhalb des polnisch-litauischen Staates und beförderten wirtschaftlichen und kulturellen Wachstum. Danzig war bekannt für seine Goldschmiede-, Uhren- und Möbelfertigung und sein reges wissenschaftliches Leben. ${ }^{10}$ Der rasch ansteigende Getreide-, Holzund sonstigen Rohstoffexport Polens nach Westeuropa und der Handel mit westeuropäischen Gewerbeerzeugnissen, für die im Lande eine wachsende Nachfrage bestand, sicherten der Stadt hohe Einkünfte. Durch die Weichsel mit ihren weitverzweigten Nebenflüssen war Danzig mit großen Gebieten Polens verbunden und vier Fünftel des polnischen Seehandels wurden über den Danziger Hafen abgewickelt. ${ }^{11}$

Der allgemeine Wohlstand nahm auch Einfluß auf die sozialen Strukturen der Bevölkerung und führte zu Ausschweifungen in der Lebenshaltung. Dafür spricht die 1455 erlassene Dan₹iger Willkür, also jene Verordnung, in der die Rechte der Stadt festgelegt waren, die unter anderem eine Kleiderordnung enthielt, die aufs Genaueste festlegte, welche Stoffarten und Schmuckstücke in welcher Menge von den einzelnen Ständen getragen werden durften. Solche Kleiderordnungen wurden insbesondere dann relevant, wenn mit aufstrebendem Wohlstand die bestehende Ordnung auseinanderzufallen drohte, weil Reichtum und Luxus zu exzessive zur Schau getragen wurden. ${ }^{12}$ Das betraf nicht nur die oberen Schichten, sondern auch Personen aus dem Volk gaben viel Geld für teure Stoffe und Luxusgüter aus. Im Laufe der Zeit verwischte sich die Abgrenzung der Stände durch die Kleidung immer mehr, weshalb nochmals 1540, also Jahrzehnte nach Hängung der Zehn Gebote-Tafel, in einer weiteren Kleiderordnung wiederum genau nachgehalten wurde, wer sich mit welchen

10 Zu nennen sind hier das Gymnasium Academicum, das Collegium Medicum, die Präsenz von Johannes Hevelius, Martin Opitz, Johannes Dantiscus.

11 Vgl. M. Bogucka, Das alte Danzig. Alltagsleben vom 15. bis 17. Jahrbundert, Leipzig 1980, S. 8.

12 Bis um die Mitte des 16. Jahrhunderts war die Mode an die burgundische Tracht angelehnt, die den Frauen schlanke Konturen verlieh und auch für die Männer enganliegende Hosen, spitze Schuhe und flandrische Mützen aus Samt vorsah. Dann wurde diese Gewandung von der sogen. spanischen Tracht abgelöst. Vgl. M. Bogucka, op. cit., S. 156. 
Artikeln in welchen Mengen und Qualitäten umgeben durfte. Erneut wurde die Kleiderfrage 1642 per Gesetz definiert.

Die Modefrage ist mit Blick auf die Zehn-Gebote-Tafel dann von Belang, wenn ihre Entstehung mit der allgemein prosperierenden Situation am Ende des 15. Jahrhunderts in Beziehung gebracht und daraus ihre Bedeutung für den sozialen Rahmen abgeleitet werden soll. Durch das Aufgreifen konkreter lokaler Bezüge wäre die Tafel dann nicht nur als allgemeine Mahnung im Zuge christlicher Tugendlehren zu lesen, sondern unmittelbar an die Danziger Bürgerschaft adressiert, die sich über ausgewiesene Details auf den Bildern wiederfinden sollte.

Adam S. Labuda untersucht nun die in den dargestellten Szenen wiedergegebene Kleidung, um sie nicht nur als Ausweis einer reichen Bevölkerung zu beschreiben, sondern auch einige für die Danziger Mode bezeichnende Stücke zu identifizieren. Hierzu gehört als herausragendes Beispiel der glockenförmig geschnittene Mantel. Gleich mehrfach findet er sich abgebildet, so auf der Darstellung in der zweiten Reihe ganz rechts, unterhalb des vierten Gebots, auf der linken Bildhälfte zum sechsten Gebot (2. Reihe links) und auch im rechten obersten Feld. Zwar sind vergleichbare Mantelarten auch für andere Regionen Europas bezeugt, doch wird speziell ein solch glöckenförmiger, aus drei ringförmig übereinander geordneten Bahnen zusammengefügter und mit einer großen Schließe zusammengehaltener Mantel als „Danziger Tracht“ bezeichnet - so auch später nachzulesen in Anton Möllers 1601 erschienenem Buch über Frauentrachten. ${ }^{13}$ Auch die Zobelmäntel und die hohen Hauben gelten als für Danzig charakteristisch. ${ }^{14}$ Hauben dieser Art finden sich beispielsweise auch auf einem 1518 in Danzig gemalten, heute im Germanischen Nationalmuseum in Nürnberg aufbewahrten Gemälde. ${ }^{15}$

Ein weiterer lokaler Bezug ist in der Schankdarstellung zu sehen, die als Negativexempel des dritten Gebots dient, das zur Einhaltung des Fei-

13 Vgl. A. S. Labuda, Kleidung als Bedeutungsträger: Zur Zehn-Gebote-Tafel aus der Marienkirche in Danzig, [in:] Bild/Geschichte. Festschrift für Horst Bredekamp, 2007, S. 420.

14 Vgl. H. Boockmann, Die Stadt im späten Mittelalter, München 1987, S. 215.

15 Vgl. P. Stieder, Ein Danziger Bildnisdiptychon von 1518, „Zeitschrift für Kunstwissenschaft", 1959, Nr. 13; R. Kahsnitz, [in:] Preußen im 19. Jahrhundert, hrsg. von U. Arnold, 1984, S. 86 ff. 
ertages anhält. Heißt es dort: „Du sollst feiern den heiligen Tag,“ wird mit entsprechendem Bildbeispiel illustriert, daß solches in Gottesdienst und Predigt besteht. Das Gegenbeispiel äußert sich nicht nur im Spruchband mit der Aufforderung zu Tanz, Trank und Spiel („,Trink, tanze, spiele, gehab dich wohl, es kommt doch wie es kommen soll' ${ }^{\circ}$, sondern auch als Mißachtung der gesetzlichen Vorschriften innerhalb der Stadt, sah doch die Danziger Willkür ein ausdrückliches Schankverbot während der Messe und an Feiertagen vor. ${ }^{16}$

Die Einbindung der Zehn Gebote in ein alltägliches Ambiente, in dem Engel und Teufel um die Seelen der Menschen ringen, entsprach der lehrhaften Intention eines verbildlichten Beichtspiegels, der unmittelbar der pastoralen Unterweisung diente. Doch die Verortung der Handlungen in eine teilweise drastische Schilderung mit unmittelbarem Zeit- und auch Ortsbezug schien angesichts der sich in Danzig zuspitzenden Situation am Ende des 15. Jahrhunderts in besonderer Weise angebracht.

Indem Labuda über die Kleidung der dargestellten Personen Bezüge zur Danziger Gesellschaft festmacht, wird deutlich, daß die in der Marienkirche ausgestellte Tafel über ihre Funktion hinaus, die biblische Lehre in anschaulicher Weise zu vermitteln, als Mahnung für ein Bürgertum gedacht war, daß im Zuge seines allgemeinen Wohlstandes drohte, die Regel und Gebote, die das soziale Zusammenleben regelten, zu übertreten. Bild für Bild werden Ausschweifungen angeprangert und mit Berufung auf die Bibel zur Einhaltung der Gesetze aufgerufen.

Vor diesem Hintergrund erklärt sich auch die Hervorhebung des Matthäus-Zitates am unteren Bildrand. Der lateinische Text bezieht sich auf die Geschichte vom reichen Jüngling, der Christus fragt, was er zu tun habe, um ins Himmelreich zu kommen (Matthäus 19, 16-26). Die Antwort Jesu (Vers 17) ist hier, etwas verkürzt, in lateinischer Fassung wiedergegeben: „Quid me interrogas de bono? unus est bonus Deus. Si autem vis ad vitam ingredi, serva mandata." ("Was heißt du mich gut? Niemand ist gut, denn der einzige Gott. Willst du zum Leben eingehen, so halte die Gebote.") Der Bezug zu den in den Bildfeldern dargestellten Zehn Geboten ist unmittelbar, denn Jesus weist ihn auf die Einhaltung

16 Vgl. P. Simson, Geschichte der Danziger Willkür, Danzig 1904 (Quellen und Darstellungen zur Geschichte Westpreußens 3), S. 50, vgl. auch S. 16 u. 24. 
der Gebote hin: „Willst du zum Leben eingehen, so halte die Gebote.“ Um aber vollkommen zu sein, rät ihm Jesus, seinen Besitz zu verkaufen und das Geld den Armen zu geben. Der ihm bleibende Schatz wird im Himmel sein (Matth 19, 21), dessen Vorgeschmack - so im Text der Tafel deutlich gemacht - darin besteht, die Gebote wie Gold zu halten. Vor diesem Hintergrund kann die antithetische Aufteilung der Bildfelder als ein Hinweis auf einen wichtigen Teil der Bergpredigt verstanden werden, nämlich jenen, wo Jesus in ebenfalls antithetischer Gegenüberstellung die Gebote wiederholt und ihnen durch seine Auslegung Nachdruck verleiht. [So heißt es beispielsweise: Ihr habt gehört, daß zu den Alten gesagt worden ist: Du sollst nicht töten, wer aber tötet, soll dem Gericht verfallen sein. Ich aber sage euch: Jeder, der seinem Bruder auch nur zürnt, soll dem Gericht verfallen sein (Matth 5, 21-48, hier 21)].

Zusammenfassend kann festgehalten werden, daß zwar die Danziger Tafel weitgehend dem geläufigen Schema dessen entspricht, was gemeinhin von Beichttafeln erwartet wird, doch über Details ihrer bildlichen Ausgestaltung wie auch dem nachstehenden Bibelzitat richtet sie sich gezielt an die Danziger Bürger und verleiht damit dem allgemeinen Appell zusätzlich einen lokal ausgerichteten Nachdruck.

\section{Die Tafel mit den Werken der Barmherzigkeit}

Geradezu ein Aufgreifen und zugleich eine Fortführung der lehrhaften Vermittlungen auf der Zehn-Gebote-Tafel scheint das 1607 von Anton Möller (1563-1611) ausgeführte Gemälde mit den Werken der Barmherzigkeit zu sein. Auch es nimmt in besonderer Weise Bezug auf die Gegebenheiten in der Stadt Danzig - und zwar in diesem Fall auf die verschiedenen nebeneinander bestehenden Glaubensrichtungen. Das Gemälde wurde vom Vorstand des Gotteskastens von St. Marien in Auftrag gegeben. ${ }^{17}$

17 Namentlich genannt sind auf der unteren Rahmenleiste Hanns Bötner, Ambrosi Kämerer, Hans Schlieff und Gerhard von Kajserswerth. Die Signatur des Malers findet sich rechts neben dem untersten der kleinen Rundbilder. 
Wie die Tafel mit den Zehn Geboten besteht auch hier die Vermittlung in Bild und Text. Der Text ist allerdings nicht mehr ins Bild integriert, sondern auf zwei separaten Tafeln unterhalb der Malerei wiedergegeben. Aufgeführt werden hier verschiedene Bibelstellen in ihrem Wortlaut wie auch mit genauem Verweis auf ihre jeweilige Quelle. Es handelt sich um Stellen aus dem Alten wie dem Neuen Testament. ${ }^{18}$

Das als Almosentafel bezeichnete Gemälde stellt in sieben medaillonartigen Bildfeldern die „Werke der Barmherzigkeit“ vor Augen. Sie sind eingeklinkt in die Verzweigungen eines Lebensbaumes, der seine Wurzeln in einer am unteren Bildrand ausgestreckt liegenden Frauengestalt hat. Durch die ihr beigegebenen Attribute - einem Holzkreuz mit Gekreuzigtem in ihrem rechten und einem Abendmahlskelch in ihrem linken Arm - ist sie als Personifikation der christlichen - und wie zu zeigen sein wird der lutheranisch-protestantischen Kirche ausgewiesen. Bestätigung findet der ikonographische Verweis durch Inschriften auf einem um ihre Taille

18 Auf der linken Tafel wird mit Versen aus Galathaer, Corinth und Jesaia die Gemeinde adressiert. Der Text ist bemerkenswerter Weise in lateinischer Sprache abgefaßt, was umso mehr auffällt, als die auf der nebenstehenden Tafel wiedergegebenen Bibelstellen aus Matth. XXV, 40, Psalm XII, 2, 3, 4 und den Sprüchen des Salomon 17 u. 19 in deutscher Sprache erscheinen. Gal 6, 5, 6: „Denn jeder wird seine eigene Bürde zu tragen haben. Wer im Evangelium unterrichtet wird, lasse seinen Lehrer an allem teilhaben, was er besitzt." Corinther 12,13 (Schreiben Paulus an die Gemeinde): Worin seid ihr denn im Vergleich mit den übrigen Gemeinden zu kurz gekommen? Höchstens darin, daß gerade ich euch nicht zur Last gefallen bin. Dann verzeiht mir dieses Unrecht. Jesaia 58, $7+8$ (Vorbereitung auf das kommende Heil): an die Hungrigen dein Brot auszuteilen, die obdachlosen Armen ins Haus aufzunehmen, wenn du einen Nackten siehst, ihn zu bekleiden und dich deinen Verwandten nicht zu entziehen. Dann wird dein Licht hervorbrechen wie die Morgenröte, und deine Wunden werden schnell vernarben. Deine Gerechtigkeit geht dir voran, die Herrlichkeit des Herrn folgt dir nach. Matth 25, 40: Darauf wird der König ihnen antworten: Amen, ich sage euch: was ihr für einen meiner geringsten Brüder getan habt, das habt ihr mir getan. Ps 41, 23, 4: Wohl dem der sich des Dürftigen annimmt, den wird der Herr erretten zur bösen Zeit. Der Herr wird ihn bewahren und beim Leben erhalten und ihm lassen wohlgehen auf Erden, und nicht geben in seiner Feinde willen. Der Her wird ihn erquicken auf seinem Siechbette. Sprüche Salomons 3, 27: Weigere dich nicht dem Dürftigen gutes zu tun, so deine Hand von Gott hat, solches zu tun. Sprüche Salomon 19, 17: Wer sich der Armen erbarmet, der leiht dem Herren, der wird ihm wieder guts vergelten. 
geschlungenen Band. Unter der groben, wohl im Rahmen einer bereits 1698 ausgeführten Restaurierung vorgenommenen Übermalung, sind in Goldlettern wiedergegeben, die Worte „Fides“ und „Caritas“ zu lesen. ${ }^{19}$ Damit ist auch die aufrechte, unverkennbar an das Schema einer stehenden Madonna angelehnte Figur bezeichnet, die hier die Caritas, also die Barmherzigkeit verkörpert. Durch die über das Schriftband wie vor allem das Wurzelwerk gezogene Verbindung zu Liegenden wird veranschaulicht, wie Barmherzigkeit aus dem rechten Glauben hervorgeht. In diesem Zusammenhang scheint es dann auch konsequent, daß die Bildmedaillons mit den guten Taten unmittelbar aus der Baumstruktur gebildet werden, ja - geradezu darin eingeschlossen sind.

Denn der rechte Glaube, der in der christlichen Kirche zu finden ist, bildet das Fundament der guten Werke. Die Bedeutung des Glaubens zu unterstreichen, findet sich zu Füßen der Liegenden ein aufgeschlagenes Buch, darin eine Darstellung des Apostel Paulus und mit einen Zitat aus einem seiner Briefen an die Galathaer $\left(6,6^{20}\right)$ eine Stelle wiedergegeben, die ausdrücklich auf den durch die Liebe tätigen Glauben hinweist. Laut Sergius Michalski wird nun durch die Berufung auf diese für das lutherische Selbstverständnis zentrale Stelle wie auch auf den Heiligen Paulus das in visueller Hinsicht altgläubig anmutende Bild im protestantischen Sinne theologisch abgesichert. ${ }^{21}$ Als zusätzlichen Beleg hierfür hat der Künstler sich bei der Paulus-Darstellung an einem Holzschnitt von Dürer orientiert. ${ }^{22}$ Anton Möller hatte schon während seiner Lehrzeit in Prag bei einem am Hofe Rudolf II. tätigen Meister Werke von Dürer kopiert und war so mit dessen künstlerischem Konzept bestens vertraut. ${ }^{23}$

19 Vgl. W. Gyssling, Anton Möller und seine Schule. Ein Beitrag zur Geschichte der niederdeutschen Renaissancemalerei, Straßburg 1917, S. 123, Anm. 52.

20 Gal 6, 6: „Der aber unterrichtet wird mit dem Wort, der theile mit allerlei Gutes, dem der ihn unterrichtet“ (Luther-Übersetzung); „,Wer im Evangelium unterrichtet wird, lasse seinen Lehrer an allem teilhaben, was er besitzt" (Einheitsbibel).

21 S. Michalski, Konfessionalisierung und Bildende Kunst, [in:] Konfessionalisierung in Ostmitteleuropa. Wirkung des religiösen Wandels im 16. und 17. Jahrbundert in Staat, Gesellschaft und Kultur, hrsg. von J. Bahlcke, A. Strohmeyer, Stuttgart 1999, S. 272.

22 Vgl. W. Gyssling, op. cit., S. 122.

23. Vgl. ibidem, S. 21 f. 
Eine im Sinne lutherischer Glaubensvorstellungen auslegbare Sicht kommt auch in der die Werke der Barmherzigkeit nach oben abschlieBenden Weltgerichtsszene zum Tragen. Das tradierte Motiv erhält bei Möller eine lutherisch-moralische Wendung. Anstatt der althergebrachten Aufteilung in Erlöste und Verdammte liegt hier der Fokus auf einer Unterscheidung von Lastern und Tugenden, die entsprechend personifiziert werden. Auf der Seite der Tugenden exponiert Möller Liebe und Fürsorglichkeit, hinterfangen von Gebet und Andacht, während auf der Seite der Laster Angst und Chaos herrschen. Über dem gnadenlos die Aufteilung vornehmenden Engel thront Christus, der mit vermittelnder Geste Gnade über Gerechtigkeit walten läßt. Die Schilderung der Liebestaten ist buchstäblich von einem Hinweis auf ihren Ursprung übertrumpft und damit gleichsam daran erinnert, daß Erlösung nicht durch gute Taten, sondern einzig durch Gott erfolgt.

Die Darstellung von Anton Möller ist aufgrund ihres ikonographischen Programms bemerkenswert, überlagern sich hier doch Motive aus unterschiedlichen Traditionszusammenhängen. Seine stehende Caritas, an der sich Einflüsse von Giorgione, Tintoretto und Palma de Vecchio abzeichnen, die allerdings weniger auf einen Italienaufenthalt Möllers zurückzuführen sind, wie Gyssling vermutet, ${ }^{24}$ sondern ihm durch niederländische Romanisten und Manieristen vermittelt wurden, ${ }^{25}$ vereint in sich mehrere ikonographische Muster. Neben Wurzel Jesse und der Personifikation von Kirche und Glauben stellt ein weiteres die Mutter Gottes mit Christuskind und Johannesknaben dar. Seine Akzentuierung erfährt dieses Motiv durch die spielenden Kinderfiguren. Da sie sich zum Teil eng an die Stehende schmiegen, einige auch unmittelbar unter ihrem Mantel Zuflucht zu suchen scheinen, schwingt auch die Vorstellung einer Schutzmantelmadonna mit. Natürlich ist der unmittelbare Bezug zur Mutter Gottes nicht zufällig, verkörpert doch gerade sie Gnade und Barmherzigkeit.

Mehr noch als der katholische Inhalt ist es die formale Angleichung an katholische Visualität, die Erstaunen erregt. Die zentrale Figur der $\mathrm{Ca}$ -

24 Vgl. Ibidem, S. $21 \mathrm{f}$.

25 Vgl. Choung-Hi Lee-Kuhn, Art. Anton Möller, in Deutsche Biographie (NDB 17, 1994, S. 637 f.). 
ritas offenbart eine geradezu demonstrative Anlehnung an das Schema der stehenden Mariafiguren. Während zwei der Putten mit kleinen Holzpferdchen spielen, fällt einer dritten, die sich an die Hand der Stehenden klammert, eine Schlange über die Schulter. Da sie gleichzeitig einem weiteren Putto die Hand zum Aufrichten reicht, klingen in dieser Motivkombination Sündenfall und Errettung an. Damit wird der Bogen zur Caritas geschlagen, die mit Maria als neuer Eva und Erlöserin der Menschheit gleichgesetzt wird. Und so windet sich auch aus ihrer Hand eine Schlange. Wurde in den Anfangsstadien der protestantischen Kunst bis etwa in die sechziger Jahre des 16. Jahrhunderts hinein das Motiv mehr oder weniger unreflektiert, einer bloßen Formgravitation folgend übernommen, muß es im Jahre 1607, auf dem Höhepunkt der reformierten Machtausbreitung in Danzig, als bewußter Bestandteil einer demonstrativen lutherischen Gegenstrategie gesehen werden.

Auffallend ist nun die Verknüpfung von Madonnenikonographie und Wurzel Jesse, ebenso wie die hier als Wurzel fungierende Frauengestalt anstelle eines männlichen Körpers, wie er dem geläufigen Muster entspräche. Diese Anspielung auf die Wurzel Jesse enthält altgläubige Züge, erscheint doch im alttestamentlichen Zusammenhang Jesse für Christus oder Maria. Die Vorstellung von Maria als virgo, virga de radice, zugleich Jungfrau, Reis der Wurzel Jesse, Mutter und Braut des Herrn und der Kirche, erfährt bei Möller ihre Umsetzung durch die als Sproß aus der Wurzel, also der Fides, hervorgehenden Caritas. Die Trennung zwischen Kirche, Glaube, Gottesmutter und Caritas ist in der Ikonographie bewußt unscharf gehalten. So ist etwa der Kelch, den die Liegende in der Hand hält, gleichermaßen Ausweis für Ecclesia, Religio, Spes wie auch Caritas. Wie das Kreuz gestaltet sich auch der Kelch als Anspielung auf Kreuzestod und Meßopfer, bleibt aber in seinem Bezug auf eine Glaubensausrichtung ambivalent.

Am Meßopfer scheiden sich schließlich auch die Geister der drei in Danzig nebeneinander bestehenden Religionen von Katholiken, Lutheranern und Reformierten. Nach der Lehre des Konzils von Trient (1545$-1563)^{26}$ sind Meßopfer und Kreuzesopfer hinsichtlich der Opfergabe

26 Das Tridentium fand in Folge des 5. Laterankonzils (1512-1517) statt und hatte ein Kirchenreform zum Ziel, bei der Dekrete über Glaubensbekenntnis, Annahme der heiligen Bücher, Ursünde, Rechtfertigung, Sakramente erlassen wurden. 
und des Opferpriesters identisch. Lediglich die Art und Weise der Darbringung ist unterschiedlich. ${ }^{27}$

Danzig nun war 1557 in einem königlichen Theologieprivileg das Abendmahl in beiderlei Gestalt gestattet worden. Und aus protestantischer Sicht war das Bild mit seinen deutlich ausgeprägt katholischen Inhalten insofern tolerabel, weil diese sich leicht im Sinne lutherischer Glaubensvorstellungen umdeuten ließen. Kehrt Luther den Glauben als einzig rechten Weg in der Religionspraxis hervor, so läßt sich dies im Bildschema in der als Fundament für alles übrige dienenden Personifikation der Fides wiederfinden. Die in den Medaillons ausformulierten Werke der Barmherzigkeit treten dem gegenüber zurück. Erstens wachsen sie aus der Wurzel heraus, sind also im übertragenen Sinne Resultate bzw. Früchte des Glaubens, zweitens ist bei einem Blickwinkel von unten auf das Bild, der not-

(Rechtfertigung = liegt komplett auf Seiten Gottes, nicht durch Ablaß, Reliquienverehrung oder andere menschliche Mühen zu erlangen; allein durch den Glauben, der Glaube wird durch das Wort bewirkt (sola gratia, sola fidses, sola scriptura, solus Christus).

27 Das genaue Verhältnis von Meß- und Kreuzesopfer wird durch repraesentatio, also der Vergegenwärtigung, memoria (Gedächtnis) und applicatio (Zuwendung) genauer bestimmt. Der Römische Katechismus (II, 4, 70) fügt noch die instauratio (Erneuerung) hinzu. Die Messe ist die lebendige, objektive Vergegenwärtigung des Kreuzesopfers in Anlehnung an die Worte Jesu Christi: „Tut dies zu meinem Gedächtnis.“ Durch die Messe wird die durch Christi Kreuzestod errungene Gnade an den Menschen weitergegeben. Der Katholische Kirche greift diese Vorstellung auf und betont, daß im Gedächtnis an den Kreuzestod auch Verkündigung und Vergegenwärtigung eingeschlossen sind. Damit unterscheidet sich der Ritus der Katholiken erheblich von dem der Protestanten, die Brot und Wein als Symbole verstehen und die Transsubstantiationslehre ablehnen. Martin Luther kritisiert das römisch-katholische Meßverständnis aufs heftigste und bezeichnet die Messe mit ihrem Opfercharakter als die größte und schrecklichste Greuel. Sich auf den Hebräerbrief (9, 27, auch Kap. 10) berufend, verweist er darauf, daß der einmalige Kreuzestod ausreicht und jedes weitere Opfer den Aussagen der Bibel widerspreche. Auch die Reformierte Kirchen sieht in Brot und Wein Zeichen für Christi Leib und Blut. Christus Ausspruch: „Die ist mein Leib“ ist laut Zwingli (1484-1531) mit „Dies bedeutet mein Leib“ zu übersetzen. Das Abendmahl gilt allein dem Gedenken an Christi Opfertod. Das Fleisch ist für den Geist ohne Nutzen, Gott handle vielmehr als Geist im Geiste des Menschen. So ist auch für Zwingli die Vorstellung von der Transsubstantiation unvorstellbar. Nach reformiertem Brauch gehören zum Abendmahl Brot und Wein, damit das Abendmahl ,unter beiderlei Gestalt“ von allen empfangen wird, weiterhin ein Abendmahlgebet, ein Dankgebet und die Einsetzungsworte. 
wendig bei einer Hängung, wie sie im Kirchenraum vorgenommen wurde, gegeben ist, die liegende Fides besser zu observieren als alles andere, was sich im weiteren nach oben anschließt.

Die auf Möllers Gemälde vorgenommene ikonographische Zusammenstellung führt auf die wechselhafte und nicht ganz unkomplizierte Entwicklung der Religionsgemeinschaften in Danzig hin. Das Gemälde läßt in dieser Form erhellende Rückschlüsse auf die Vorstellungen vom rechten Glauben zu, die seit der Reformation heftig diskutiert wurden und sich in der Besetzung der einzelnen Kirchen mit unterschiedlich ausgerichteten Gemeinden niederschlugen.

Obwohl die Lehre von den guten Werken mit zahlreichen Stellen aus der Bibel zu begründen ist, war sie spätestens seit der Reformation Gegenstand theologischer Auseinandersetzungen. Martin Luther, der 1520 den Sermon von den guten Werken verfaßt hatte, polemisierte gegen die römische Werkgerechtigkeit, also die Auffassung, man könne sich vor Gott durch gute Taten rechtfertigen. Das Tridentinische Konzil hingegen hielt in einem Dekret von 1547 fest, daß der im Glauben Stehende durch Werke der Barmherzigkeit Gnade erlangen könne.

In Anton Möllers Almosentafel wird die Bedeutung der guten Werke zur Gnadenerlangung deutlich hervorgehoben. Dabei sind die in den Medaillons ausgeführten guten Taten durch das sie rahmende Astwerk fest an den Stamm der Caritas angebunden, die wiederum im Glauben wurzelt. Auch erweist sich in diesem Zusammenhang die enge Anlehnung an die Marienikongraphie als absichtsvoll, verkörpert doch Maria Gnade und Barmherzigkeit zugleich. Auf Möllers Darstellung wird diese Funktion durch die mütterliche Hinwendung zu den Kindern unterstrichen.

Es bleibt festzuhalten, daß Möllers Tafel mit den Werken der Barmherzigkeit mehrere ikonographische Konzepte vereint, deren jedes sich mit einer spezifischen Glaubensausrichtung in Verbindung bringen läßt. Überwiegen die an die katholische Lehre angelehnten Einflüsse, obwohl das Gemälde in einem Kirchenraum hängt, der zu diesem Zeitpunkt zwar ausschließlich von der Lutheranischen Gemeinde genutzt wurde, so ist dem entgegenzuhalten, daß sich diese noch wenige Jahrzehnte zuvor den Ort mit den Katholiken geteilt hatte, mit deren Vorstellungen das Bild konform war. Von 1557 bis 1572 hielten sowohl die evangelischaugsburgischen als auch die katholische Gemeinde ihre Gottesdienste in 
der Marienkirche ab. Da die Lutheraner sich der Kirchenkunst gegenüber weitgehend tolerant zeigten, bot ihnen die katholische Kirchenausstattung keinen Anstoß. Trotz Einführung der Reformation blieb sie weiterhin im Kirchenraum erhalten und wurde sogar den Bedürfnissen der lutherischen Gemeinde entsprechend weiterverwendet. Solch merkwürdige Aufteilung unter den von deutlichen Unterschieden in der Glaubensauffassung getrennten Gemeinden ist lediglich damit zu begründen, daß beide sich gegen die immer stärker werdende Übermacht der Reformierten Kirche durchzusetzen hatten. Im Bestreben, die Frage nach der Wahl des rechten Weges gegen die reformierte Prädestinationslehre zu stärken, gingen die Danziger Lutheraner mitunter so weit, eine Position einzunehmen, die der katholischen Werkgerechtigkeitslehre nahekam. ${ }^{28}$ Und Möllers Bild ließ sich zudem auch hinsichtlich der lutheranischen Glaubensfokussierung ausdeuten. Die besondere Stellung der lutherisch-protestantischen Kirche erklärt sich wiederum aus der Geschichte der Stadt.

Danzig hat innerhalb der polnischen Geschichte schon von alters her eine Sonderstellung eingenommen. Hin- und hergerissen zwischen Deutschem Orden, Preußischem Bund und Polen blieb die Positionierung der Stadt in der politischen Landschaft nicht ohne Einfluß auf die Entwicklung der religiösen Gemeinschaften. So kann gerade Danzig allerhand konfessionelle Bewegung und Veränderungen verzeichnen.

Als Möller sein Gemälde erstellte, standen drei Konfessionen mehr oder weniger gleichberechtigt nebeneinander und alle drei führten umfangreiche und komplizierte kulturpolitische Auseinandersetzungen, zu denen neben der des Kunstmäzenatentums vor allem auch die nach der Rechtmäßigkeit der religiösen Kunst, also die Bilderfrage gehörte.

Seit den dreißiger Jahren des 16. Jahrhunderts dominierten in Danzig zwar die Lutheraner, während außerhalb der Stadt die Katholiken vorherrschten, die dann - angeführt von karrierebewußten Angehörigen des polnischen Adels - nach 1600 zunehmend nach Danzig vordrangen. Gleichzeitig waren seit 1580 wichtige Positionen in der Stadt von Angehörigen des Calvinismus besetzt, die bis kurz nach 1600 beständig an Macht gewannen, bis ihnen schließlich 1612 durch die Lutheraner Einhalt geboten wurde. Im gleichen Jahr wies der Rat der Stadt die Jesuiten aus Danzig.

$28 \quad$ Vgl. S. Michalski, op. cit., S. 271. 
Welche Dimension die Religionsstreitigkeiten entfalteten, zeigt sich unter anderem daran, daß nicht nur das Bildprogramm in den Kirchen, sondern ebenso in den weltlichen Räumen der Stadt davon beeinflußt wurde, etwa im Artushof und im Rechtsstädtischen Rathaus. ${ }^{29}$

Die Tafel mit den Werken der Barmherzigkeit entstand also zu einem Zeitpunkt, als die Calvinisten auf dem Höhepunkt ihrer Macht waren. Die Marienkirche sah sich förmlich umzingelt von reformierten Glaubensgemeinschaften und bildete innerhalb der Stadt eine letzte und einzige Bastion lutherischen Glaubens, die sich aber zunehmend vom Eindringen calvinistischer Glaubensanhänger eingeengt sah. Zumindest sprechen die vielen bilderlosen Schriftepitaphien in der Kirche für calvinistische Auftraggeber. ${ }^{30}$ Doch ist hier auch zu bedenken, daß gerade in Danzig in der dritten Dekade des Jahrhunderts der Typ des barocken, dekorativen Inschriftenepitaphs aufkam, das keine konfessionelle Grenze mehr kannte. Nichts desto trotz galt es gegenüber den Calvinisten den lutherisch-evangelischen Glauben zu stärken, ohne dabei zu sehr Stellung gegen die Katholiken zu beziehen, zu denen immer noch eine größere Nähe bestand als zu den Calvinisten. Die Bildwerke, wie überhaupt die religiöse Kunst, dienten der lutherischen Auffassung nach dem Gedächtnis und der Erinnerung und unterstützten darin die christliche Lehre. Möllers Darstellung ist der Versuch, die Haltung der lutherisch-evangelischen Konfession in einer Weise zu veranschaulichen, die ihre Toleranz hinsichtlich der Bilderfrage deutlich werden läßt.

So unterschiedlich beide Gemälde - also die Tafel mit den Zehn Geboten und die mit den Werken der Barmherzigkeit - und so unterschiedlich die Zeitpunkte ihrer Entstehung auch sind, so ist ihnen doch gemeinsam, daß sie jeweils zu Zeiten in Auftrag gegeben wurden, die von gesellschaftsverändernder Relevanz waren. Beide Bilder suchten den Bürgern der Stadt einen Spiegel entgegenzuhalten, der sie an ihre Pflichten als gute Christen gemahnte. Ging es um 1480/90 um allgemein als christlich erachtete, die vor allem für das alltägliche Miteinander von Bedeutung wa-

29 Ibidem, S. $268 \mathrm{ff}$.

30 K. Cieślak, Kościót - cmentarzem. Sz̨tuka nagrobna w Gdańsku (XV-XVIII w.), Gdańsk 1992, S. 32, 59-69, zit. bei S. Michalski, op. cit., S. 270. 
ren, suchte das Gemälde von Anton Möller zur rechten Haltung gegenüber der konfessionellen Entscheidung aufzurufen.

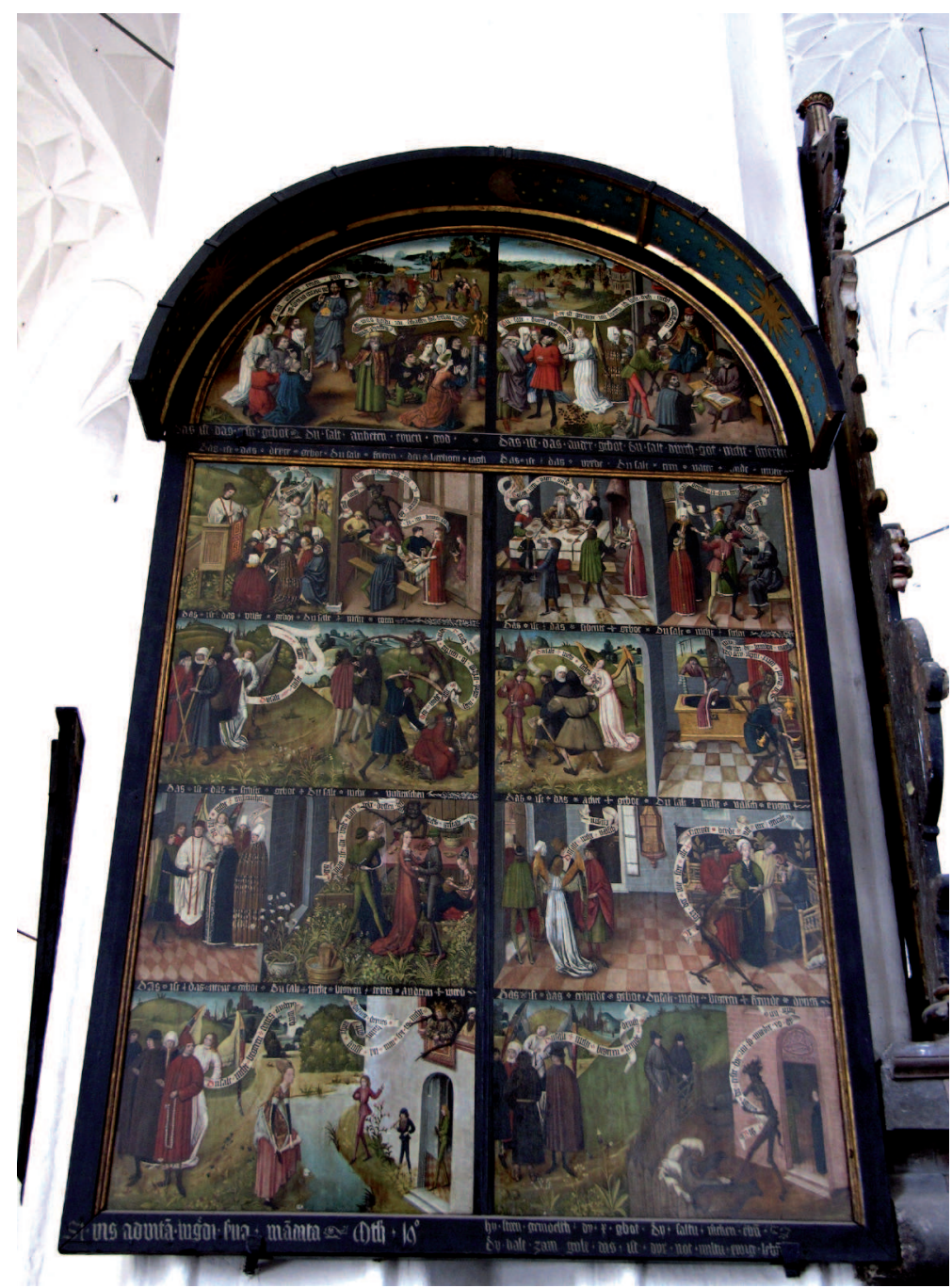

Il. 1. Die Tafel der Zehn Gebote, Marienkirche, Danzig (fot. V. Hildebrand-Schat) 


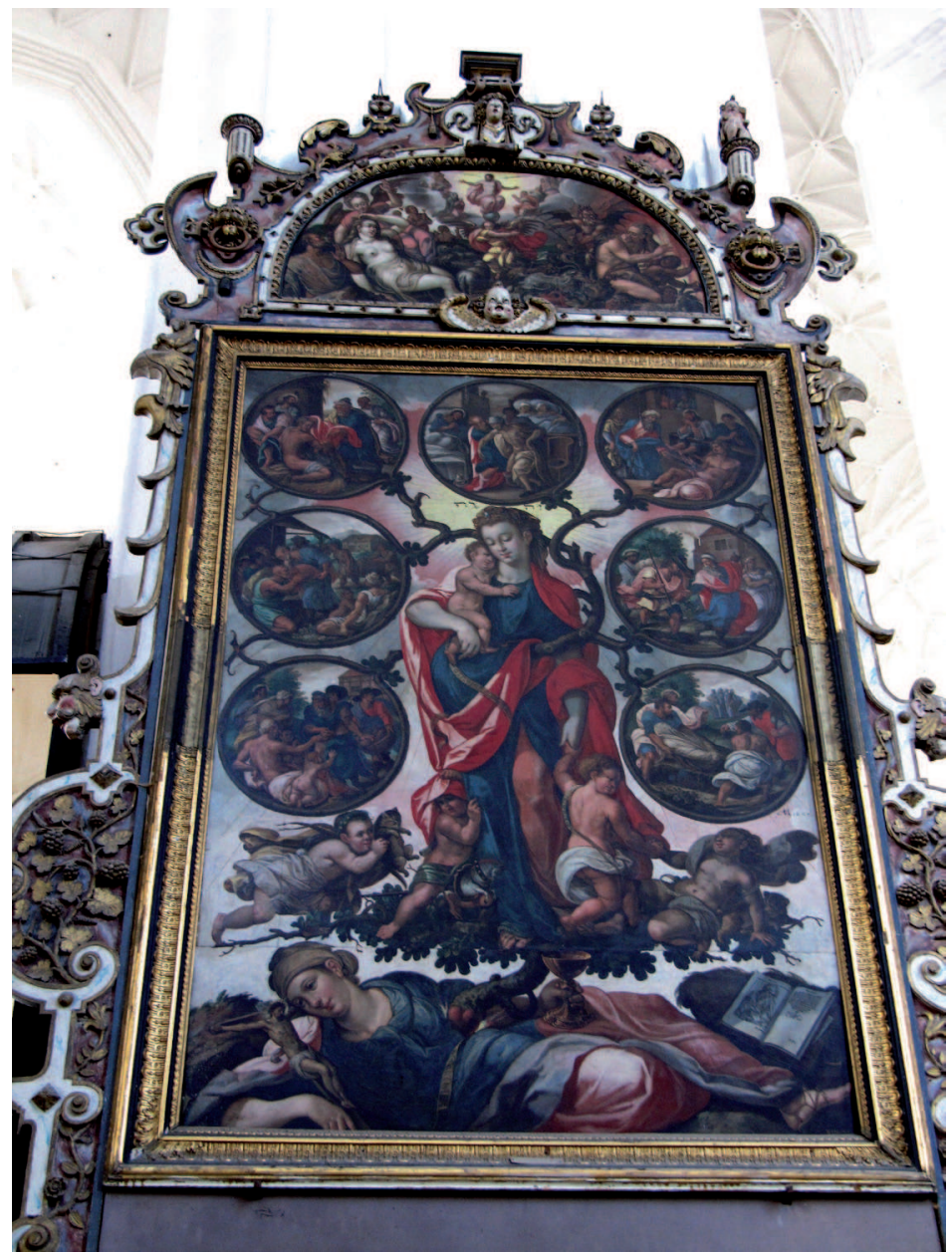

Il. 2. Die Tafel mit den Werken der Barmherzigkeit, Marienkirche, Danzig (fot. V. Hildebrand-Schat)

\section{Summary}

The representation of commandments in the Church of St. Mary in Danzig

Entering the church of St. Mary in Danzig the visitor will be struck by the two paintings in the north-western wing not far away from the high altar. Even though 
related though their subject they date form different periods. One was provided by a Netherland master between 1480 and 1490, the other from the regional artist Anton Möller in 1607. Both paintings can be summarized under the term "instructive," because they address the viewer with his duties as a good Christian. The painting form the end of the $15^{\text {th }}$ century shows the Ten Commandments, the painting from Anton Möller the seven works of charity. With that both paintings are part of instruction as it follows form Christian religion. Remarkable however is the interrelation both paintings show to social-historical events of their time. This can be observed by details included in the representation. The painting with the Ten Commandments gives a close observation of the population form Danzig showing the different roles within society. They evidence of wealth and prosperity, which took influence on morality. Therefore the clerical became aware of the necessity to remind of the duties as they were given with the Ten Commandments. The works of charity allude to the religious constellations, which has become competing powers at the turn of the century. The painting of Möller results to be a sort of manifest for the Lutheran Church against the Reformed Church. The Calvinist at that time had powerful positions within the municipality and took more and more influence on the political development. Therefore the Protestant were interested to demonstrate the power they had, especially when they allied with the Catholics. Such both paintings are of an importance, which exceed the clerical context by far. While both representations refer to the current situation of their time they can be considered as a document of the social and political development. 